Results Following the implementation of the direct entry pathway in May 2010 the CTBT for all patients admitted direct to our hospital have reduced. This is statistically significant when looking at Quarter 2 results from baseline. Patient safety has not been compromised. Patients who were admitted directly have been asked about their experience and if anything could be done differently from their perspective. They have said:

- The process is quick which is good from their perspective

- They are fully informed

- The ambulance crews deal with them competently

- The lab staff are waiting for their arrival.

Conclusions The CCU nurses have embraced this development and expansion of their nursing practice, allowing major changes to be made to the Primary Angioplasty pathway within the existing infrastructure, despite the challenges of working within the complex nature of traditional geographical referral patterns. Along with the work of all members of the multi disciplinary team this has significantly reduced times to treatment for patients.

\section{SCREENING FIRST DEGREE RELATIVES FOR HYPERTROPHIC CARDIOMYOPATHY: 12-MONTH EXPERIENCE OF A CARDIO- GENETICS NURSE SERVICE}

doi:10.1136/heartjnl-2011-300198.77

\author{
${ }^{1} \mathrm{~S}$ Finch, ${ }^{2} \mathrm{~S}$ Russell, ${ }^{1} \mathrm{D}$ Kumar, ${ }^{1} \mathrm{Z}$ R Yousef. ${ }^{1}$ University Hospital of Wales, Cardiff, UK; \\ ${ }^{2}$ Wales Heart Research Institute, Cardiff, UK
}

Introduction Hypertrophic cardiomyopathy (HCM) is an autosomally transmitted cardiomyopathy with an estimated gene prevalence of 1:500, and an important cause of sudden cardiac death. Screening to identify at risk first degree relatives is therefore recommended. The British Heart Foundation (BHF) recently funded nine Nationwide cardio-genetic nurses to support local initiatives. Our application for a nurse was successful and we present our 12-month experience of HCM screening.

Methods We mapped the course of patients with suspected HCM referred to our tertiary heart muscle clinic which serves a population of 1.4 million. Following phenotype confirmation, a family tree and contact details from the index case were recorded by the cardiogenetic nurse. The index case was given literature to pass onto at risk relatives. The information pack included an open invitation (referral via primary care) to attend for screening. For relatives residing outside our catchment area screening was arranged via links with the BHF cardio-genetic network and other health care providers. Relatives domiciled outside UK were given our details with offers to support screening. Throughout, strict adherence to patient confidentiality was maintained.

Results Over 12 months, 64 index HCM cases presented to our heart muscle clinic. Pedigree analysis identified 221 first degree relatives at risk of carrying the HCM gene; mean index-to-at RR: 1-to-3.4 (range 0-14 subjects). Of the 221 at risk subjects, 71 (19 through paediatrics) have undergone screening through clinical assessment at our unit with plans for long-term 2-5 yearly follow-up in view of variable gene penetrance. Of the 71 screened subjects, 15 were newly diagnosed with HCM. Newly diagnosed HCM patients underwent further risk stratification for sudden cardiac death; where we identified 3 patients at high risk $(\geq 2$ conventional high sudden death risk factors). After appropriate counselling, these 3 patients have received primary prevention defibrillators. Despite our approach, 52 subjects remain unscreened (Abstract 77 table 1), either due to complex family relationships $(n=14)$, personal preference $(n=28)$ and/or geographical/logistical reasons $(n=10)$.
Abstract 77 Table 1 Screening outcomes of 221 at risk subjects identified from 64 index cases of hypertrophic cardiomyopathy

\begin{tabular}{ll}
\hline & Number of Patients \\
\hline New screening initiated (local heart muscle clinic) & 52 \\
New screening initiated (local paediatric clinic) & 19 \\
New screening initiated (out of area service) & 6 \\
Pre-existing screening in place & 63 \\
Personal preference (declined screening) & 28 \\
Awaiting response from subject (literature delivered) & 19 \\
Complex family relationships (unable to deliver literature) & 14 \\
Geographical/Logistical constraints & 10 \\
Subject deceased (non-hypertrophic cardiomyopathy) & 3 \\
Subject deceased (hypertrophic cardiomyopathy) & 7 \\
\hline
\end{tabular}

Conclusions Proactive screening for HCM can be effectively facilitated by cardio-genetic nurse services. Each new index case generates 3-4 at risk relatives who require long-term surveillance. Of 71 asymptomatic at risk subjects screened in our unit, we diagnosed 15 new cases of HCM, and 3 patients at high risk of sudden cardiac death who subsequently received primary prevention defibrillator implantation.

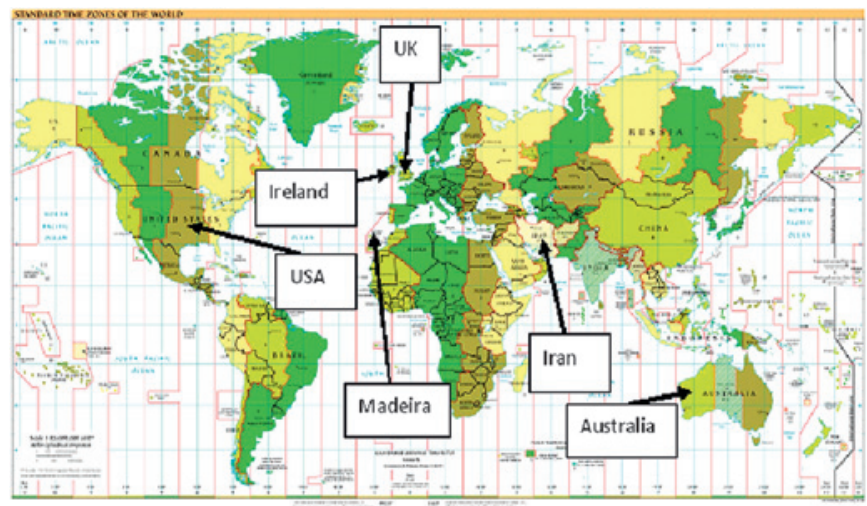

Abstract 77 Figure 1

\section{FIRST YEAR EXPERIENCE OF A DEDICATED "RADIAL LOUNGE" FOR PATIENTS UNDERGOING ELECTIVE PERCUTANEOUS CORONARY PROCEDURES}

doi:10.1136/heartjnl-2011-300198.78

S Brewster, R Weerackody, K Khimdas, A Little, N Cleary, A Penswick, M Rothman, A Archbold. London Chest Hospital, London, UK

Introduction The potential to achieve safe early mobilisation and same day discharge on a consistent basis after radial artery access has provided us with the opportunity to make a step change in the way we deliver elective care to patients undergoing percutaneous coronary procedures. We designed a dedicated "radial lounge" to accommodate patients before and after their procedure with the aim of minimising the feeling of "hospitalisation" that accompanies most encounters with health services. The lounge is a day case unit that has no beds, only chairs, and televisions but no cardiac monitors. Patients remain in their clothes throughout their hospital visit. Here we report our first year's experience of this facility. Methods: The study population comprised all patients who attended the radial lounge between July 2009-June 2010 for coronary angiography or percutaneous coronary intervention (PCI). Patients were suitable for the radial lounge if they were elective cases who had a satisfactory radial pulse and no pre-procedure contraindication to 
same day discharge. Patients were excluded if they had any of the following: an unsuitable radial pulse, planned femoral access, prior coronary artery bypass surgery, or the requirement for an overnight hospital stay for planned complex/high risk PCI, renal impairment, or social reasons. The final decision regarding route of arterial access was left to the operator.

Results In the one year study period, 1548 patients were managed in the radial lounge. 1109 patients underwent coronary angiography, $114(10.2 \%)$ of whom also had a pressure wire or intravascular ultrasound, and 439 underwent PCI. This represented approximately $88 \%$ of our unit's elective angiograms and $60 \%$ of our elective PCIs. Among the patients who underwent angiography, 938 (84.5\%) were performed radially and 1076 (97.0\%) were discharged from the radial lounge on the same day as their procedure. Among the PCI patients, 359 (81.8\%) were performed radially and 372 $(84.7 \%)$ were discharged the same day. The PCI group included 326 $(74.3 \%)$ patients who had a single vessel treated, 105 (23.9\%) who had two vessels or a bifurcation with a significant side branch treated, and $8(1.8 \%)$ patients who had three vessels treated. There were no deaths or arrhythmias in the radial lounge. Requirement for overnight admission was significantly more common after femoral access compared with radial access for both angiography (4.1\% vs $2.8 \% ; \mathrm{p}<0.05)$ and PCI (21.3\% vs $14.2 \% ; \mathrm{p}<0.01)$

Conclusions A dedicated radial lounge free of cardiac monitors is a safe environment in which to manage most patients before and after elective coronary angiography and PCI. The lack of monitoring necessitates patient selection but this does not prevent the lounge being suitable for the majority of elective patients. Femoral access is associated with a significantly greater requirement for overnight admission.

\section{REMAINING CLOTHED FOR RADIAL DIAGNOSTIC CORONARY ANGIOGRAPHY: AN IMPROVEMENT IN THE PATIENT JOURNEY}

doi:10.1136/heartjnl-2011-300198.79

S Eve, M Sinha, T A Wells. Salisbury District Hospital, Salisbury, UK

Background Patients undergoing invasive diagnostic coronary angiography (DCA) for the first time often display high levels of anxiety at the time of their procedure as they are unfamiliar with the cardiac catheter laboratory set up. It is therefore part of the cardiac catheter laboratory staff's role to reduce patient fears and hence improve their journey through the cardiac catheter laboratory. Several Cardiac centres have recently introduced radial lounges whereby patients feel less "hospitalised" by not needing to undress for their procedure.

Methods Following infection control approval, between mid-August 2010 and the end of October 2010, patients undergoing radial DCA at Salisbury District Hospital were offered the option of remaining clothed for their procedure. Each patient was given an information leaflet included in which was explained possible downsides to being dressed including if CPR were needed then clothes would be cut, failure of radial access and the subsequent need for femoral access, and the possibility of soiling the clothes with either blood or iodine. The only caveat stated was that female patients were not allowed to wear an underwire bra. Following their DCA, patients were then asked to fill in an anonymous questionnaire in which they were asked about their experience and whether not having to undress made them feel more relaxed.

Results 57 consecutive patients underwent (DCA) during this time period $(100 \%$ uptake) with an average age of $68.1 \pm 9.6$ years. $71 \%$ were male and $21 \%(12 / 57)$ had undergone a DCA previously. Of these 12 patients $92 \%(11 / 12)$ stated that not having to undress was a good idea while an identical number felt much more relaxed than their previous DCA experience. Of the 45 patients that had not had a previous DCA, $96 \%(43 / 45)$ stated that not having to undress was a good idea while $96 \%$ (43/45) felt that this had made them feel very relaxed during their pathway. The other two patients felt that it made no difference. No patients required cross-over to femoral access and there were no blood or iodine stains on any clothes. Having patients remain dressed did not reduce fluoroscopic image quality and there were no issues with infection.

Conclusion Offering patients the option of having their radial DCA done without undressing is safe and helps to improve the patient journey through the cardiac catheter laboratory by making them feel more relaxed and less hospitalised. This is now standard at our Institution.

\section{PPCI: IS THERE A ROLE FOR THE ACS ANP?}

doi:10.1136/heartjnl-2011-300198.80

V Oriolo, J Tagney. Bristol Heart Institute University Hospital Bristol NHS FT, Bristol, UK

Introduction Primary Percutaneous Coronary Intervention (PPCI) is now considered the treatment of choice for patients experiencing ST Elevation Myocardial Infarction (STEMI) (European Society of Cardiology/European Association for Cardio-Thoracic Surgery 2010). One of the many benefits claimed is reduced length of stay due to decreased morbidity (Zahn et al 2000, Kalla et al 2006). To assess performance of one English PPCI 24/7 provider organisation against the national average length of stay (LOS) for patients post PPCI, a retrospective baseline audit was conducted. This demonstrated an average LOS of 4.4 days which is above the National Infarct Angioplasty Project 2008 average LOS of 3 days. A 48 h nurse led discharge (NLD) protocol was therefore developed and introduced by the acute coronary syndromes advanced nurse practitioner to streamline the patient journey. This instructed the nurse and/or physician to ensure appropriate investigation and documentation was carried out in a timely manner to avoid unnecessary delays in patient discharge.

Method Suitability criteria for the 48-h NLD protocol were established, which included: absence of acute complications (eg, bleeding, haemodynamic instability, ongoing chest pain, ejection fraction $<40 \%$, respiratory compromise); appropriate support at discharge. Following the baseline audit, data were electronically collected prospectively for 5 months, measuring date of admission to date of discharge to the usual place of residence. After 5 months the audit was repeated to assess the average length of stay for patients presenting with STEMI.

Result Between 1st April 2010 and 31st August 2010, a total of 274 patients were admitted with STEMI. Of these, 122 (45\%) met the NLD criteria and were discharged by the ACS ANP. The remaining $152(55 \%)$ were discharged by the medical physicians. It was noted that introduction of the protocol also facilitated a structured approach to discharge for the medical team. The average LOS for all PPCI patients $(n=274)$ decreased from 4.4 days to 3 days $(30 \%)$. For patients that were seen and discharged solely by the advanced nurse practitioner $(n=122)$, the average LOS decreased from 4.4 days to 2.0 days $(55 \%)$

Conclusions/Implications In the current financial climate, a decrease in LOS can have a significant impact on any organisational resources thus increasing efficiency saving and patient throughput. This demonstrates part of the added value the advanced nurse practitioner brings to patient care and to tertiary centres that provide a 24/7 PPCI service. 